\title{
A Bivariate Index for Visually Measuring Marginal Inhomogeneity in Square Tables
}

\author{
Shuji Ando ${ }^{1}$ \\ ${ }^{1}$ Department of Information and Computer Technology, Faculty of Engineering, Tokyo University of Science, Japan \\ Correspondence: Shuji Ando, Department of Information and Computer Technology, Faculty of Engineering, Tokyo \\ University of Science, Katsushika-ku, Tokyo, 125-8585, Japan. E-mail: shuji.ando@ @rs.tus.ac.jp
}

Received: June 27, 2019 Accepted: August 5, 2019 Online Published: August 15, 2019

doi:10.5539/ijsp.v8n5p58 URL: https://doi.org/10.5539/ijsp.v8n5p58

\begin{abstract}
For square tables, the marginal homogeneity model which has a structure that the row marginal distribution is equal to the column marginal distribution was proposed. Thereafter, various extended models of marginal homogeneity have been proposed, these models can be classified into two types marginal inhomogeneity. On the other hand, various indexes which measure the degree of deviation from marginal homogeneity have been proposed. However these indexes cannot concurrently define degrees of deviation from marginal homogeneity with respect to two types marginal inhomogeneity. This paper proposes a bivariate index that can concurrently define degrees of deviation from those. The proposed bivariate index would also be utility for visually comparing degrees of deviation from marginal homogeneity in several tables using confidence regions.
\end{abstract}

Keywords: confidence region, power divergence, marginal homogeneity, ordered category, visualization

\section{Introduction}

Square tables having the same ordinal classifications are generally obtained by two times repeated measurement or matched pair data for categorical variables in clinical study and in the study of social mobility. Consider the data in Tables 1a and 1b taken from Gane, Deray, Liaw, Lim, Lai, Rasenack, Wang, Papatheodoridis, Bisceglie, Buti, Samuel, Uddin, Bosset, and Trylesinski (2014). In the intent-to-treat population of the study, 680 patients with chronic hepatitis $\mathrm{B}$ were randomized in the telbivudine treatment group and 687 patients with chronic hepatitis $\mathrm{B}$ were randomized in the lamivudine treatment group. The first time (baseline) and second time (end of study) of estimate glomerular filtration rate (eGFR) were classified into 3 categories: $<60 \mathrm{~mL} / \mathrm{min} / 1.73 \mathrm{~m}^{2}, 60-89 \mathrm{~mL} / \mathrm{min} / 1.73 \mathrm{~m}^{2}$, and $\geq 90 \mathrm{~mL} / \mathrm{min} / 1.73 \mathrm{~m}{ }^{2}$, respectively. The eGFR categories indicate that " $\geq 90$ " is best category and " $<60$ " is worst category.

Table 1 . The two tables below are square tables of eGFR category at end of study by treatment groups, stratified by eGFR category at baseline; source Gane et al. (2014)

(a) Telbivudine group

\begin{tabular}{ccccc}
\hline & \multicolumn{3}{c}{ Baseline } & \\
\cline { 2 - 4 } End of study & $\geq 90$ & $60-89$ & $<60$ & Total \\
\hline$\geq 90$ & 382 & 185 & 1 & 568 \\
$60-89$ & 36 & 71 & 4 & 111 \\
$<60$ & 1 & 0 & 0 & 1 \\
\hline Total & 419 & 256 & 5 & 680 \\
\hline
\end{tabular}

(b) Lamivudine group

\begin{tabular}{ccccc}
\hline & \multicolumn{3}{c}{ Baseline } & \\
\cline { 2 - 4 } End of study & $\geq 90$ & $60-89$ & $<60$ & Total \\
\hline$\geq 90$ & 366 & 123 & 2 & 491 \\
$60-89$ & 78 & 106 & 4 & 188 \\
$<60$ & 1 & 5 & 2 & 8 \\
\hline Total & 445 & 234 & 8 & 687 \\
\hline
\end{tabular}

For above data, we are interested in checking whether or not the marginal distribution of first time is equal to the marginal distribution of second time. As the analysis of homogeneity of marginal probabilities, the marginal homogeneity model which has a structure that the row marginal distribution is equal to the column marginal distribution was proposed by Stuart (1955). Thereafter, various extended models of marginal homogeneity have been proposed, these models can be classified into two types marginal inhomogeneity. One is the extended marginal homogeneity model which was proposed by Tomizawa (1984). Second is the marginal logit model which was considered by McCullagh (1977). Furthermore extended models for the extended marginal homogeneity model or the marginal logit model have been proposed (see, e.g., Tahata and Tomizawa (2008) and Kurakami, Tahata and Tomizawa (2013)). On the other hand, various indexes that measure the degree of deviation from marginal homogeneity have been proposed (see, for example, Tomizawa, Miyamoto and Ashihara (2003) and Iki, Tahata and Tomizawa (2012)). However these existing indexes cannot concurrently define 
the degree of deviation from marginal homogeneity corresponding to the extended marginal homogeneity model and the marginal logit model. We believe that for comparing degrees of deviation from marginal homogeneity in Tables A and B, it is important that the index can concurrently define the degree of deviation from those. Because, the index corresponding to the extended marginal homogeneity model may show that the degree of deviation from marginal homogeneity in Table A is bigger than that in Table B, but the index corresponding to the marginal logit model may show that the degree of deviation from marginal homogeneity in Table B is bigger than that in Table A. We will show to exist the above situation using the artificial data. For measuring the degree of deviation from marginal homogeneity, we propose a bivariate index that can concurrently define the degree of deviation from marginal homogeneity corresponding to the extended marginal homogeneity model and the marginal logit model.

\section{Existing Models and Indexes for Marginal Homogeneity}

\subsection{Notation and Existing Models}

Consider a square table having the same ordinal classifications. Let $p_{i j}$ denote the cell probability in the $i$ th row and $j$ th column of the table $(i=1, \ldots, R ; j=1, \ldots, R)$. The marginal homogeneity model, which was proposed by Stuart (1955), is defined as follows

$$
\pi_{i \cdot}=\pi_{\cdot i} \quad \text { for } i=1, \ldots, R,
$$

where $\pi_{i \cdot}=\sum_{t=1}^{R} \pi_{i t}$ and $\pi_{\cdot i}=\sum_{s=1}^{R} \pi_{s i}$. The marginal homogeneity model can be expressed using cumulative marginal probabilities as follows

$$
F_{i}^{X}=F_{i}^{Y} \quad \text { for } i=1, \ldots, R-1,
$$

where $F_{i}^{X}=\sum_{s=1}^{i} \pi_{s}$ and $F_{i}^{Y}=\sum_{t=1}^{i} \pi_{t \cdot}$. Since the cumulative marginal probabilities $\left\{F_{i}^{X}\right\}$ and $\left\{F_{i}^{Y}\right\}$ contain the same cell probability (for example, main diagonal cell probabilities), we introduce an another cumulative probability excluding overlapped cell probabilities as follows

$$
G_{1(i)}=\sum_{s=1}^{i} \sum_{t=i+1}^{R} \pi_{s t} \quad \text { and } \quad G_{2(i)}=\sum_{s=i+1}^{R} \sum_{t=1}^{i} \pi_{s t} .
$$

Using the cumulative probabilities $\left\{G_{1(i)}\right\}$ and $\left\{G_{2(i)}\right\}$, the marginal homogeneity model can also be expressed as follows

$$
G_{1(i)}=G_{2(i)} \quad \text { for } i=1, \ldots, R-1 .
$$

Moreover, the marginal homogeneity model can be expressed as follows

$$
H_{1(i)}=H_{2(i)} \quad \text { for } i=1, \ldots, R-1,
$$

where $H_{1(i)}=F_{i}^{X}\left(1-F_{i}^{Y}\right)$ and $H_{2(i)}=\left(1-F_{i}^{X}\right) F_{i}^{Y}$. Thus, the marginal homogeneity model can be expressed using various cumulative probabilities.

Thereafter, extended models of marginal homogeneity have been proposed. The extended marginal homogeneity model, which was proposed by Tomizawa (1984), is defined as follows

$$
G_{1(i)}=\tau G_{2(i)} \quad \text { for } i=1, \ldots, R-1 .
$$

This model is an extended model based on the form (2.3). A special case of this model obtained by putting $\tau=1$ is equivalent to the marginal homogeneity model. The marginal logit model, which was proposed by McCullagh (1977), is defined as follows

$$
H_{1(i)}=\theta H_{2(i)} \quad \text { for } i=1, \ldots, R-1 .
$$

This model is an extended model based on the form (2.4). A special case of this model obtained by putting $\theta=1$ is equivalent to marginal homogeneity model. Thereafter, the furthermore extension of extended marginal homogeneity and marginal logit models were proposed by Tahata and Tomizawa (2008) and Kurakami et al. (2013), respectively.

For measuring goodness-of-fit of the model, test statistics are usually used. The family of power divergence test statistics or $\phi$-divergence test statistics contain well known the Pearson's chi-squared statistic or likelihood ratio statistic as special cases (see, Read and Cressie 1988, Pardo, 2006). We do not focus on measuring goodness-of-fit of the marginal homogeneity model, are interested in comparing degrees of deviation from marginal homogeneity. When the marginal 
homogeneity model does not hold for several tables, we are interested in comparing degrees of deviation from it. However, it is inappropriate for comparing degrees of deviation from marginal homogeneity in several tables using test statistics because test statistics depend on sample size and dimension $R$. When two data underlying same cell probability $p_{i j}$ have different sample size, test statistics values unequal although degrees of deviation from the model are same. For comparing degrees of deviation from marginal homogeneity in several tables, we are interested in an index value that does not depend on sample size and dimension $R$.

\subsection{Index $\phi^{(\lambda)}$ for Measuring Marginal Inhomogeneity Using Cumulative Probabilities of (2.3)}

Tomizawa et al. (2003) proposed the index $\phi^{(\lambda)}$ that measures the degree of deviation from marginal homogeneity. The index $\phi^{(\lambda)}$ does not depend on sample size and dimension $R$. Assuming that $G_{1(i)}+G_{2(i)}>0$ for $i=1, \ldots, R-1$, the index $\phi^{(\lambda)}$ is defined as follows:

$$
\phi^{(\lambda)}=\frac{\lambda(\lambda+1)}{2^{\lambda}-1} I_{G}^{(\lambda)} \quad(\lambda>-1),
$$

where

$$
I_{G}^{(\lambda)}=\frac{1}{\lambda(\lambda+1)} \sum_{i=1}^{R-1}\left[G_{1(i)}^{*}\left\{\left(\frac{G_{1(i)}^{*}}{q_{i}^{*}}\right)^{\lambda}-1\right\}+G_{2(i)}^{*}\left\{\left(\frac{G_{2(i)}^{*}}{q_{i}^{*}}\right)^{\lambda}-1\right\}\right],
$$

with

$$
G_{1(i)}^{*}=\frac{G_{1(i)}}{\delta}, \quad G_{2(i)}^{*}=\frac{G_{2(i)}}{\delta}, \quad q_{i}^{*}=\frac{G_{1(i)}^{*}+G_{2(i)}^{*}}{2}, \quad \delta=\sum_{i=1}^{R-1}\left(G_{1(i)}+G_{2(i)}\right) .
$$

Note that $I_{G}^{(\lambda)}$ is the power divergence between $\left\{G_{1(i)}^{*}, G_{2(i)}^{*}\right\}$ and $\left\{q_{i}^{*}, q_{i}^{*}\right\}$ for $i=1, \ldots, R-1$, and for $\lambda \rightarrow 0 I_{G}^{(0)}$ is KullbackLeiblar information between them. The index $\phi^{(\lambda)}$ has the following characteristics: (1) $\phi^{(\lambda)}$ lies between 0 and 1 ; (2) $\phi^{(\lambda)}=0$ if and only if the marginal homogeneity model holds; and (3) $\phi^{(\lambda)}=1$ if and only if the degree of deviation from marginal homogeneity is maximal (namely, for $i=1, \ldots, R-1$ either $G_{1(i)}=0$ or $G_{2(i)}=0$ ).

Under the extended marginal homogeneity model (2.5), the index $\phi^{(\lambda)}$ can be simply (as a function of parameter $\tau$ ) expressed as

$$
\phi^{(\lambda)}=\frac{1}{2^{\lambda}-1}\left[2^{\lambda}\left\{\left(\frac{\tau}{\tau+1}\right)^{\lambda+1}+\left(\frac{1}{\tau+1}\right)^{\lambda+1}\right\}-1\right] \quad(\lambda>-1) .
$$

Thus, we see that the index $\phi^{(\lambda)}$ can measure the degree of deviation from marginal homogeneity corresponding to the extended marginal homogeneity model. On the other hand, under the marginal logit model (2.6), the index $\phi^{(\lambda)}$ cannot be simply (as a function of parameter $\theta$ ) expressed.

\subsection{Index $\psi^{(\lambda)}$ for Measuring Marginal Inhomogeneity Using Cumulative Probabilities of (2.4)}

Iki et al. (2012) proposed the index $\psi^{(\lambda)}$ that measures the degree of deviation from marginal homogeneity. The index $\psi^{(\lambda)}$ does not depend on sample size and dimension $R$. Assuming that $H_{1(i)}+H_{2(i)}>0$ for $i=1, \ldots, R-1$, the index $\psi^{(\lambda)}$ is desined as follows:

where

$$
\psi^{(\lambda)}=\frac{\lambda(\lambda+1)}{2^{\lambda}-1} I_{H}^{(\lambda)} \quad(\lambda>-1),
$$

$$
I_{H}^{(\lambda)}=\frac{1}{\lambda(\lambda+1)} \sum_{i=1}^{R-1}\left[H_{1(i)}^{*}\left\{\left(\frac{H_{1(i)}^{*}}{Q_{i}^{*}}\right)^{\lambda}-1\right\}+H_{2(i)}^{*}\left\{\left(\frac{H_{2(i)}^{*}}{Q_{i}^{*}}\right)^{\lambda}-1\right\}\right],
$$

with

$$
H_{1(i)}^{*}=\frac{H_{1(i)}}{\Delta}, \quad H_{2(i)}^{*}=\frac{H_{2(i)}}{\Delta}, \quad Q_{i}^{*}=\frac{H_{1(i)}^{*}+H_{2(i)}^{*}}{2}, \quad \Delta=\sum_{i=1}^{R-1}\left(H_{1(i)}+H_{2(i)}\right) .
$$

Note that $I_{H}^{(\lambda)}$ is the power divergence between $\left\{H_{1(i)}^{*}, H_{2(i)}^{*}\right\}$ and $\left\{Q_{i}^{*}, Q_{i}^{*}\right\}$ for $i=1, \ldots, R-1$, and for $\lambda \rightarrow 0 I_{H}^{(0)}$ is Kullback-Leiblar information between them. The index $\psi^{(\lambda)}$ has the following characteristics: (1) $\psi^{(\lambda)}$ lies between 0 and 1 , (2) $\psi^{(\lambda)}=0$ if and only if the marginal homogeneity model holds, and (3) $\psi^{(\lambda)}=1$ if and only if the degree of deviation from marginal homogeneity is maximal (namely, for $i=1, \ldots, R-1$ either $H_{1(i)}=0$ or $H_{2(i)}=0$ ).

Under the marginal logit model (2.6), the index $\psi^{(\lambda)}$ can be simply (as a function of parameter $\theta$ ) expressed as

$$
\psi^{(\lambda)}=\frac{1}{2^{\lambda}-1}\left[2^{\lambda}\left\{\left(\frac{\theta}{\theta+1}\right)^{\lambda+1}+\left(\frac{1}{\theta+1}\right)^{\lambda+1}\right\}-1\right] \quad(\lambda>-1) .
$$


Thus, we see that the index $\psi^{(\lambda)}$ can measure the degree of deviation from marginal homogeneity corresponding to the marginal logit model. On the other hand, under the extended marginal homogeneity model (2.5), the index $\psi^{(\lambda)}$ cannot be simply (as a function of parameter $\tau$ ) expressed.

\subsection{Other Indexes for Measuring Marginal Inhomogeneity}

For square tables with ordered categories, some indexes that measure the degree of deviation from marginal homogeneity have been proposed other than indexes $\phi^{(\lambda)}$ and $\psi^{(\lambda)}$. For example, Tahata, Tajima and Tomizawa (2006), Tahata, Iwashita and Tomizawa (2006), and Tahata, Iwashita and Tomizawa (2008). Under the extended marginal homogeneity model or the marginal logit model, these indexes cannot be simply (as a function of parameter $\tau$ or $\theta$ ) expressed such as the indexes $\phi^{(\lambda)}$ and $\psi^{(\lambda)}$. Thus, these indexes cannot measure the degree of deviation from marginal homogeneity corresponding to the extended marginal homogeneity model or the marginal logit model.

\section{Bivariate Index and Confidence Region}

We propose a bivariate index that can concurrently define the degree of deviation from marginal homogeneity corresponding to the extended marginal homogeneity model and the marginal logit model. The proposed bivariate index would also be utility for visually comparing degrees of deviation from marginal homogeneity in several tables using confidence regions.

\subsection{Definition of the Bivariate Index}

Assuming that $G_{1(i)}+G_{2(i)}>0$ and $H_{1(i)}+H_{2(i)}>0$ for $i=1, \ldots, R-1$, we propose the bivariate index

$$
\Phi^{(\lambda)}=\left(\begin{array}{c}
\phi^{(\lambda)} \\
\psi^{(\lambda)}
\end{array}\right) ; \text { a } 2 \times 1 \text { vector. }
$$

It has the properties: (1) $\Phi^{(\lambda)}=(0,0)^{\prime}$ if and only if the marginal homogeneity model holds, (2) $\Phi^{(\lambda)}=(1,1)^{\prime}$ if and only if the degree of deviation from marginal homogeneity is maximal, in the sense that $G_{1(i)}=0$ and $H_{1(i)}=0$ (then $G_{2(i)}>0$ and $H_{2(i)}>0$ ) or $G_{2(i)}=0$ and $H_{2(i)}=0$ (then $G_{1(i)}>0$ and $H_{1(i)}>0$ ) for $i=1, \ldots, R-1$. Note that the structure of maximum degree of deviation from marginal homogeneity for the proposed bivariate index $\Phi^{(\lambda)}$ is different from that for indexes $\phi^{(\lambda)}$ and $\psi^{(\lambda)}$. The proposed bivariate index is constructed based on similar idea to Ando, Tahata and Tomizawa (2017) and Ando, Tahata and Tomizawa (2019). Ando et al. (2017) and Ando et al. (2019) proposed bivariate indexes corresponding to the model that is different the marginal homogeneity model.

Indexes $\phi^{(0)}$ and $\psi^{(0)}$ indicate the minimum Kullback-Leiblar information between two distribution, respectively. However, when $\lambda \neq 0$, indexes $\phi^{(\lambda)}$ and $\psi^{(\lambda)}$ cannot expressed as the minimum power divergence. Therefore, if the user wants to measure the degree of deviation from marginal homogeneity by using the minimum power divergence between two distribution, then the user should use the bivariate index $\Phi^{(0)}$.

\subsection{A Confidence Region for the Bivariate Index}

Let

$$
\begin{aligned}
& n=\left(n_{11}, n_{12}, \ldots, n_{1 R}, n_{21}, n_{22}, \ldots, n_{2 R}, \ldots, n_{R 1}, n_{R 2}, \ldots, n_{R R}\right)^{\prime} ; \text { a } R^{2} \times 1 \text { vector } \\
& \pi=\left(\pi_{11}, \pi_{12}, \ldots, \pi_{1 R}, \pi_{21}, \pi_{22}, \ldots, \pi_{2 R}, \ldots, \pi_{R 1}, \pi_{R 2}, \ldots, \pi_{R R}\right)^{\prime} ; \text { a } R^{2} \times 1 \text { vector. }
\end{aligned}
$$

Assuming that $n$ is according to a multinomial distribution $\operatorname{Multi}(N ; \pi)$ with sample size $N\left(=\sum \sum n_{i j}\right)$. Then $\sqrt{N}(\hat{\pi}-\pi)$ is according to asymptotically a $R^{2}$-dimensinal normal distribution with mean zero vector and covariance matrix $\operatorname{Diag}(\pi)-$ $\pi \pi^{\prime}$, where $\hat{\pi}=n / N$ and $\operatorname{Diag}(\pi)$ is diagonal matrix (see, e.g., Agresti, 2013, p. 590). To estimate the indexes, $\hat{\phi}^{(\lambda)}$ and $\hat{\psi}^{(\lambda)}$ are obtained by $\phi^{(\lambda)}$ and $\psi^{(\lambda)}$ with $\left\{\pi_{i j}\right\}$ replaced by $\left\{\hat{\pi}_{i j}\right\}$, respectively, where $\hat{\pi}_{i j}=n_{i j} / N$. The $\widehat{\Phi}^{(\lambda)}$ is obtained by $\Phi^{(\lambda)}$ with $\phi^{(\lambda)}$ and $\psi^{(\lambda)}$ replaced by $\hat{\phi}^{(\lambda)}$ and $\hat{\psi}^{(\lambda)}$, respectively. Let $\left(\partial \Phi^{(\lambda)} / \partial \pi^{\prime}\right)$ be the $2 \times R^{2}$ matrix that the entry in row $k$ and column $l$ is $\partial \Phi_{k}^{(\lambda)}(\pi) / \partial \pi_{l}$, where $\Phi_{1}^{(\lambda)}$ and $\Phi_{2}^{(\lambda)}$ are $\phi^{(\lambda)}$ and $\psi^{(\lambda)}$, respectively, and $\pi_{l}$ is the lth element of $\pi$. When $N$ approaching infinity, the estimated bivariate index can be approximated by

$$
\widehat{\Phi}^{(\lambda)}=\Phi^{(\lambda)}+\left(\frac{\partial \Phi^{(\lambda)}}{\partial \pi^{\prime}}\right)(\hat{\pi}-\pi)+o(\|\hat{\pi}-\pi\|),
$$

where $o(\|\hat{\pi}-\pi\|)$ converges to $(0,0)^{\prime}$. Using the delta method (see, Agresti, 2013, Sec. 16.1), $\sqrt{N}\left(\widehat{\Phi}^{(\lambda)}-\Phi^{(\lambda)}\right)$ has asymptotically a bivariate normal distribution with mean zero vector and covariance matrix

$$
\Sigma=\left(\frac{\partial \Phi^{(\lambda)}}{\partial \pi^{\prime}}\right)\left(\operatorname{Diag}(\pi)-\pi \pi^{\prime}\right)\left(\frac{\partial \Phi^{(\lambda)}}{\partial \pi^{\prime}}\right)^{\prime}=\left(\begin{array}{ll}
\sigma_{11} & \sigma_{12} \\
\sigma_{21} & \sigma_{22}
\end{array}\right),
$$


with $\sigma_{12}=\sigma_{21}$. The elements $\sigma_{11}, \sigma_{12}$ and $\sigma_{22}$ are expressed as follows:

$$
\begin{aligned}
& \sigma_{11}=\left(\frac{\partial \phi^{(\lambda)}}{\partial \pi^{\prime}}\right)\left(\operatorname{Diag}(\pi)-\pi \pi^{\prime}\right)\left(\frac{\partial \phi^{(\lambda)}}{\partial \pi^{\prime}}\right)^{\prime}=\frac{1}{\delta^{2}} \sum_{s=1}^{R-1} \sum_{t=s+1}^{R}\left[\pi_{s t}\left(v_{s t}^{(\lambda)}\right)^{2}+\pi_{t s}\left(w_{t s}^{(\lambda)}\right)^{2}\right] \\
& \sigma_{12}=\left(\frac{\partial \phi^{(\lambda)}}{\partial \pi^{\prime}}\right)\left(\operatorname{Diag}(\pi)-\pi \pi^{\prime}\right)\left(\frac{\partial \psi^{(\lambda)}}{\partial \pi^{\prime}}\right)^{\prime}=\frac{1}{\delta \Delta} \sum_{s=1}^{R-1} \sum_{t=s+1}^{R}\left[\pi_{s t} v_{s t}^{(\lambda)} V_{s t}^{(\lambda)}+\pi_{t s} w_{t s}^{(\lambda)} V_{t s}^{(\lambda)}\right], \\
& \sigma_{22}=\left(\frac{\partial \psi^{(\lambda)}}{\partial \pi^{\prime}}\right)\left(\operatorname{Diag}(\pi)-\pi \pi^{\prime}\right)\left(\frac{\partial \psi^{(\lambda)}}{\partial \pi^{\prime}}\right)^{\prime}=\frac{1}{\Delta^{2}}\left[\sum_{s=1}^{R} \sum_{t=1}^{R} \pi_{s t}\left(V_{s t}^{(\lambda)}\right)^{2}\right],
\end{aligned}
$$

where for $\lambda \neq 0$,

$$
\begin{aligned}
v_{s t}^{(\lambda)}= & \frac{2^{\lambda}}{2^{\lambda}-1}\left[\sum_{i=s}^{t-1}\left\{\left(G_{1(i)}^{c}\right)^{\lambda}+\lambda\left(\left(G_{1(i)}^{c}\right)^{\lambda}-\left(G_{2(i)}^{c}\right)^{\lambda}\right) G_{2(i)}^{c}\right\}-(t-s) \frac{\left(2^{\lambda}-1\right) \phi^{(\lambda)}+1}{2^{\lambda}}\right], \\
w_{t s}^{(\lambda)}= & \frac{2^{\lambda}}{2^{\lambda}-1}\left[\sum_{i=s}^{t-1}\left\{\left(G_{2(i)}^{c}\right)^{\lambda}+\lambda\left(\left(G_{2(i)}^{c}\right)^{\lambda}-\left(G_{1(i)}^{c}\right)^{\lambda}\right) G_{1(i)}^{c}\right\}-(t-s) \frac{\left(2^{\lambda}-1\right) \phi^{(\lambda)}+1}{2^{\lambda}}\right], \\
V_{s t}^{(\lambda)}= & \frac{2^{\lambda}}{2^{\lambda}-1}\left[\frac{\left(2^{\lambda}-1\right)\left(1-\psi^{(\lambda)}\right)}{2^{\lambda}} \sum_{m=1}^{R-1}\left(\frac{\partial H_{1(m)}}{\partial \pi_{s t}}+\frac{\partial H_{2(m)}}{\partial \pi_{s t}}\right)\right. \\
& \left.-\sum_{i=1}^{R-1}\left[\frac{\partial H_{1(i)}}{\partial \pi_{s t}}\left\{1-\left(H_{1(i)}^{c}\right)^{\lambda}-\lambda H_{2(i)}^{c}\left(\left(H_{1(i)}^{c}\right)^{\lambda}-\left(H_{2(i)}^{c}\right)^{\lambda}\right)\right\}+\frac{\partial H_{2(i)}}{\partial \pi_{s t}}\left\{1-\left(H_{2(i)}^{c}\right)^{\lambda}-\lambda H_{1(i)}^{c}\left(\left(H_{2(i)}^{c}\right)^{\lambda}-\left(H_{1(i)}^{c}\right)^{\lambda}\right)\right\}\right]\right],
\end{aligned}
$$

and for $\lambda=0$,

$$
\begin{aligned}
& v_{s t}^{(0)}=\frac{1}{\log 2}\left[\sum_{i=s}^{t-1} \log G_{1(i)}^{c}-(t-s)\left(\phi^{(0)}-1\right) \log 2\right], \\
& w_{t s}^{(0)}=\frac{1}{\log 2}\left[\sum_{i=s}^{t-1} \log G_{2(i)}^{c}-(t-s)\left(\phi^{(0)}-1\right) \log 2\right], \\
& V_{s t}^{(0)}=\frac{1}{\log 2}\left[\log 2\left(1-\psi^{(0)}\right) \sum_{m=1}^{R-1}\left(\frac{\partial H_{1(m)}}{\partial \pi_{s t}}+\frac{\partial H_{2(m)}}{\partial \pi_{s t}}\right)+\sum_{i=1}^{R-1}\left(\frac{\partial H_{1(i)}}{\partial \pi_{s t}} \log H_{1(i)}^{c}+\frac{\partial H_{2(i)}}{\partial \pi_{s t}} \log H_{2(i)}^{c}\right)\right],
\end{aligned}
$$

with

$$
\begin{array}{cc}
G_{1(i)}^{c}=\frac{G_{1(i)}}{G_{1(i)}+G_{2(i)}}, \quad G_{2(i)}^{c}=\frac{G_{2(i)}}{G_{1(i)}+G_{2(i)}}, & H_{1(i)}^{c}=\frac{H_{1(i)}}{H_{1(i)}+H_{2(i)}}, \quad H_{2(i)}^{c}=\frac{H_{2(i)}}{H_{1(i)}+H_{2(i)}}, \\
\frac{\partial H_{1(i)}}{\partial \pi_{s t}}=\sum_{k=1}^{i} \sum_{l=i+1}^{R}\left\{I(s=k) \pi_{\cdot t}+I(t=l) \pi_{s}\right\}, & \frac{\partial H_{2(i)}}{\partial \pi_{s t}}=\sum_{k=i+1}^{R} \sum_{l=1}^{i}\left\{I(s=k) \pi_{\cdot t}+I(t=l) \pi_{s}\right\}
\end{array}
$$

and where $I(\cdot)$ is the indicator function, $I(\cdot)=1$ if true, 0 if not. Note that the asymptotic variances $\sigma_{11}$ and $\sigma_{22}$ of $\phi^{(\lambda)}$ and $\psi^{(\lambda)}$, respectively, have been shown by Tomizawa et al. (2003) and Iki et al. (2012).

An approximate $100(1-\alpha) \%$ confidence region of the bivariate index $\Phi^{(\lambda)}$ is derived by

$$
N\left(\widehat{\Phi}^{(\lambda)}-\Phi^{(\lambda)}\right)^{\prime} \widehat{\Sigma}^{-1}\left(\widehat{\Phi}^{(\lambda)}-\Phi^{(\lambda)}\right) \leq \chi_{(1-\alpha ; 2)}^{2}
$$

where $\chi_{(1-\alpha ; 2)}^{2}$ is the $1-\alpha$ quantile of the chi-square distribution with two degrees of freedom and $\widehat{\Sigma}$ is obtained by $\Sigma$ with $\left\{\pi_{i j}\right\}$ replaced by $\left\{\hat{\pi}_{i j}\right\}$. 


\section{Numerical Examples}

In this Secttion, using numerical examples we will show that the proposed bivariate index $\Phi^{(\lambda)}$ is utility.

\subsection{The Utility of Concurrently Characterizing}

For comparing degrees of deviation from marginal homogeneity in two Tables, it is important that the index can concurrently define degrees of deviation from marginal homogeneity corresponding the extended marginal homogeneity model and the marginal logit model. We will show the above importance using the artificial data such as Tables $2 \mathrm{a}$ and $2 \mathrm{~b}$. These data are $4 \times 4$ square tables with $n=1000$ each. For these data, using the index $\phi^{(0)}$ we can judge that the degree of deviation from marginal homogeneity in Table $2 \mathrm{~b}$ is bigger than that in Table $2 \mathrm{a}$, using the index $\psi^{(0)}$ we can judge that the degree of deviation from marginal homogeneity in Table 2a is bigger than that in Table 2b. Thus, for comparing degrees of deviation from marginal homogeneity in two Tables, the conclusion may change by using which of indexes $\phi^{(\lambda)}$ and $\psi^{(\lambda)}$. On the other hand, using the index $\Phi^{(0)}$, from Figure 1, we can judge that there is no the magnitude relationship between the degree of deviation from marginal homogeneity in Table $2 a$ and that in Table $2 b$. Thus, for comparing degrees of deviation from marginal homogeneity, we suggest that the analyst should use the proposed bivariate index $\Phi^{(\lambda)}$.

Table 2. Artificial square tables; $n=1000$ for each

\begin{tabular}{cccc} 
(a) & & & \\
\hline 101 & 58 & 85 & 95 \\
33 & 114 & 45 & 65 \\
23 & 43 & 96 & 71 \\
26 & 31 & 32 & 82
\end{tabular}

\begin{tabular}{cccc} 
(b) & & & \\
\hline 196 & 24 & 38 & 38 \\
8 & 188 & 27 & 27 \\
5 & 10 & 206 & 19 \\
2 & 6 & 6 & 200 \\
\hline
\end{tabular}

Table 3. Estimates of $\phi^{(0)}$ and $\psi^{(0)}$, approximate standard errors for $\hat{\phi}^{(0)}$ and $\hat{\psi}^{(0)}$, and approximate $95 \%$ confidence intervals for $\phi^{(0)}$ and $\psi^{(0)}$, for the data of Tables $2 \mathrm{a}$ and $2 \mathrm{~b}$

\begin{tabular}{|c|c|c|c|}
\hline & $\begin{array}{l}\text { Estimated } \\
\text { index }\end{array}$ & $\begin{array}{l}\text { Standard } \\
\text { error }\end{array}$ & $\begin{array}{l}\text { Confidence } \\
\text { interval }\end{array}$ \\
\hline \multicolumn{4}{|c|}{ (a) For Table 2a } \\
\hline$\phi^{(0)}$ & 0.1467 & 0.0265 & $(0.0947,0.1987)$ \\
\hline$\psi^{(0)}$ & 0.0968 & 0.0175 & $(0.0626,0.1311)$ \\
\hline \multicolumn{4}{|c|}{ (b) For Table $2 \mathrm{~b}$} \\
\hline$\phi^{(0)}$ & 0.4108 & 0.0621 & $(0.2891,0.5324)$ \\
\hline$\psi^{(0)}$ & 0.0314 & 0.0062 & $(0.0193,0.0435)$ \\
\hline
\end{tabular}

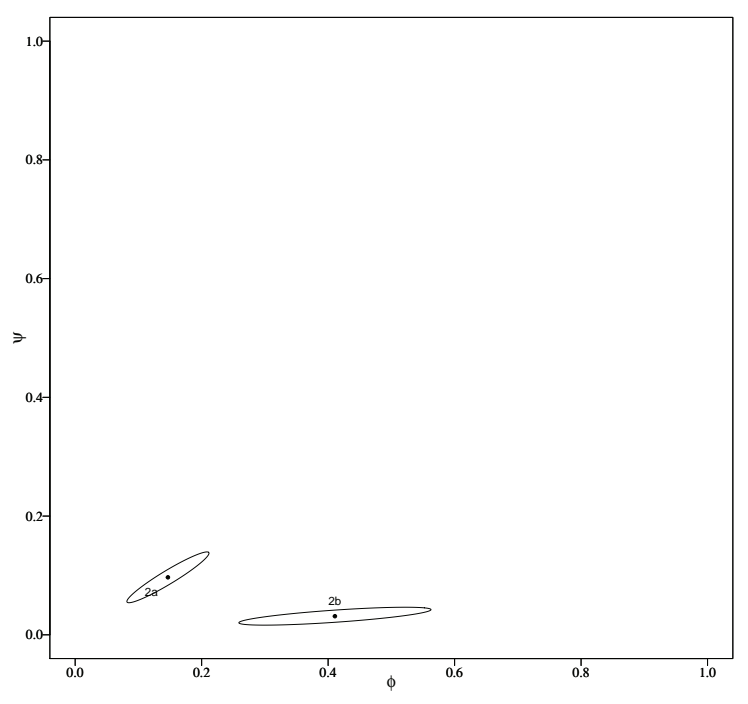

Figure 1. Approximate $95 \%$ confidence regions for $\Phi^{(0)}$, for the data of Tables $2 \mathrm{a}$ and $2 \mathrm{~b}$ 


\subsection{Example of Real Data}

For the data in Tables 1a and 1b, we are interested in whether patients in the telbivudine group are better than patients in the lamivudine group about the change from baseline to end of study in eGFR category as a clinical interest. Generally, when there is the treatment effect, the marginal homogeneity model does not hold. Thus, it can be assumed the bigger the degree of deviation from marginal homogeneity, the higher the treatment effect.

We shall compare degrees of deviation from marginal homogeneity in Tables $1 \mathrm{a}$ and $1 \mathrm{~b}$ using the confidence regions for $\Phi^{(0)}$. The estimates of $\Phi^{(0)}$ are

$$
\widehat{\Phi}^{(0)}=\left(\begin{array}{l}
0.3517 \\
0.2072
\end{array}\right) \text { and } \widehat{\Phi}^{(0)}=\left(\begin{array}{l}
0.0349 \\
0.0162
\end{array}\right)
$$

respectively, and the estimates of $\Sigma$ are

$$
\widehat{\Sigma}=\left(\begin{array}{ll}
2.3317 & 1.1678 \\
1.1678 & 0.8108
\end{array}\right) \quad \text { and } \quad \widehat{\Sigma}=\left(\begin{array}{ll}
0.3125 & 0.1428 \\
0.1428 & 0.0674
\end{array}\right)
$$

respectively. From Figure 2, we can see that the degree of deviation from marginal homogeneity in Table 1a is bigger than that in Table $1 \mathrm{~b}$, and patients in the telbivudine group are better than patients in the lamivudine group about the change from baseline to end of study in eGFR category.

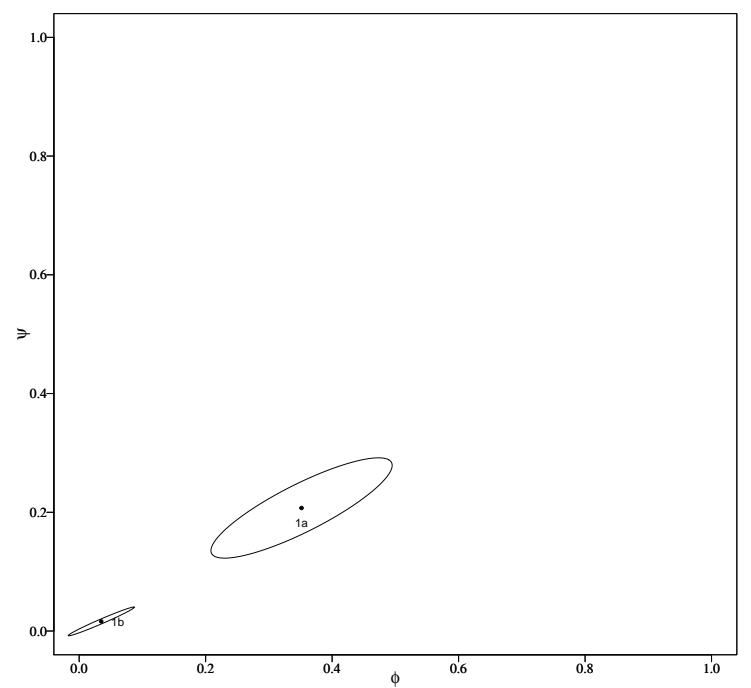

Figure 2. Approximate $95 \%$ confidence regions for $\Phi^{(0)}$, for the data of Tables $1 \mathrm{a}$ and $1 \mathrm{~b}$

\section{Concluding Remarks}

This paper proposed a bivariate index which can concurrently define degrees of deviation from marginal homogeneity corresponding to the extended marginal homogeneity model and the marginal logit model. We believe that it is important to concurrently define degrees of deviation from marginal homogeneity corresponding to the extended marginal homogeneity model and the marginal logit model. Because, (1) for comparing degrees of deviation from marginal homogeneity in two Tables, the conclusion may change by using which of indexes $\phi^{(\lambda)}$ and $\psi^{(\lambda)}$ as shown in Sectction 4.1, and (2) indexes $\phi^{(\lambda)}$ and $\psi^{(\lambda)}$ are not independent as shown in Secttion 3.2. In addition, the proposed bivariate index would be utility for visually comparing degrees of deviation from marginal homogeneity using confidence regions. For comparing degrees of deviation from marginal homogeneity in several square contingency tables, we believe that the proposed bivariate index produces results that are easier to interpret than existing indexs.

\section{Acknowledgements}

The author is greatly thankful to reviewer and the editor for the comments and suggestions to improve this article. The author also thanks Mr. S. Ishikawa for his utility comments. 


\section{References}

Agresti, A. (2013). Categorical Data Analysis, 3rd edition. New Jersey: Wiley.

Ando, S., Tahata, K., \& Tomizawa, S. (2017). Visualized measure vector of departure from symmetry for square contingency tables. Statistics in Biopharmaceutical Research, 9, 212-224. https://doi.org/10.1080/19466315.2016.1258006

Ando, S., Tahata, K., \& Tomizawa, S. (2019). A bivariate index vector for measuring departure from double symmetry in square contingency tables. Advances in Data Analysis and Classification, 13, 519-529. https://doi.org/10.1007/s11634-018-0320-7

Gane J. E., Deray, G., Liaw, F. Y., Lim, G. S., Lai, L. C., Rasenack, J., ... \& Trylesinski, A. (2014). Telbivudine improves renal function in patients with chronic hepatitis B. Gastroenterology, 146, 138-146.

Iki, K., Tahata, K., \& Tomizawa, S. (2012). Measure of departure from marginal homogeneity using marginal odds for multi-way tables with ordered categories. Journal of Applied Statistics, 39, 279-295. https://doi.org/10.1080/02664763.2011.586682

Kurakami, H., Tahata, K., \& Tomizawa, S. (2013). Generalized marginal cumulative logistic model for multi-way contingency tables. SUT Journal of Mathematics, 49, 19-32.

McCullagh, P. (1977). A logistic model for paired comparisons with ordered categorical data. Biometrika, 64, 449-453. https://doi.org/10.2307/2345320

Pardo, L. (2006). Statistical Inference Based on Divergence Measures. New York: Chapman \& Hall.

Read, T. R. C., \& Cressie, N. A. C. (1988). Goodness-of-Fit Statistics for Discrete Multivariate Data. New York: Springer. https://doi.org/10.1007/978-1-4612-4578-0

Stuart, A. (1955). A test for homogeneity of the marginal distributions in a two-way classification. Biometrika, 42, 412-416. https://doi.org/10.2307/2333387

Tahata, K., Iwashita, T., \& Tomizawa, S. (2006). Measure of departure from symmetry of cumulative marginal probabilities for square contingency tables with ordered categories. SUT Journal of Mathematics, 42, 7-29. https://doi.org/10.1080/02331880802190521

Tahata, K., Iwashita, T., \& Tomizawa, S. (2008). Measure of departure from conditional marginal homogeneity for square contingency tables with ordered categories. Statistics, 42, 453-466.

Tahata, K., Tajima, K., \& Tomizawa, S. (2006). A measure of asymmetry of marginal ridits for square contingency tables with ordered categories. Journal of the Japanese Society of Computational Statistics, 19, 69-85. https://doi.org/10.5183/jjscs1988.19.69

Tahata, K., \& Tomizawa, S. (2008). Generalized marginal homogeneity model and its relation to marginal equimoments for square contingency tables with ordered categories. Advances in Data Analysis and Classification, 2, 295-311.

Tomizawa, S. (1984). Three kinds of decompositions for the conditional symmetry model in a square contingency table. Journal of the Japan Statistical Society, 14, 35-42.

Tomizawa, S., Miyamoto, N., \& Ashihara, N. (2003). Measure of departure from marginal homogeneity for square contingency tables having ordered categories. Behaviormetrika, 30, 173-193.

\section{Copyrights}

Copyright for this article is retained by the author(s), with first publication rights granted to the journal.

This is an open-access article distributed under the terms and conditions of the Creative Commons Attribution license (http://creativecommons.org/licenses/by/4.0/). 